\title{
Recursos y alternativas de tratamiento para los minerales de teluro de Sonora $\left(\right.$ México) ${ }^{(\bullet)}$
}

\author{
S. Aguayo ${ }^{(*)}$, E. Pérez ${ }^{(* *)}$ y M.A. Encinas ${ }^{(*)}$ \\ Resumen El teluro es un elemento escaso, pero con un espectro amplio de aplicaciones. Su producción está \\ limitada a la obtenida por el tratamiento de los lodos electrolíticos de las refinerías de cobre. De las \\ fuentes alternativas de teluro, los telururos de metales preciosos son atractivos por la asociación natu- \\ ral de estos dos elementos, con la posibilidad de obtener el teluro como subproducto. Sonora (Méxi- \\ co), se caracteriza por ser una zona rica en teluros con más de treinta especies registradas. Este artí- \\ culo describe las principales localizaciones en que se encuentran minerales de teluro en Sonora \\ (México), señalando sus principales características, así como las especies hasta ahora registradas. Se \\ analizan, además, las posibles rutas de extracción y recuperación acuosa de teluro, de acuerdo con la \\ fisicoquímica de los sistemas teluro-metales preciosos. \\ Palabras clave: Procesamiento acuoso de teluros. Recursos y procesamiento de telururos. \\ Tratamiento de telururos.

\section{Occurency and aqueous processing of tellurides from Sonora (México)}

\begin{abstract}
Tellurium production is limited mainly to that obtained from the treatment of electrolyte muds from copper refineries. However, there are several other sources from which the precious metal tellurides are potentially attractive. This work presents a review of the main localities in Sonora (México), where tellurides have been found. In addition, based upon the physical chemistry fundamentals for tellurium and precious metal tellurides, the aqueous extraction and recovery routes are disccused.
\end{abstract}

Keywords: Precious metal tellurides ocurrency and aqueous processing. Aqueous processing of tellurides. Tellurides treatment.

\section{INTRODUCCIÓN}

El teluro es un elemento escaso en la naturaleza. $\mathrm{Su}$ concentración promedio en la corteza terrestre es del orden de 0,002 ppm; similar en abundancia al platino y más escaso que el selenio. En contraposición, y debido probablemente a su comportamiento geoquímico, parecido al del azufre, es un elemento que se combina formando una extensa variedad de minerales, de los cuales se han descrito formalmente más de 160 especies.

$\mathrm{Al}$ igual que otros elementos menores, el teluro se recupera a partir de productos intermedios que se obtienen durante el procesamiento de minerales de sulfuros no férreos. Actualmente, se dispone de otras fuentes potencialmente importantes, tales como residuos de procesos de extracción por disolventes para

\footnotetext{
$(\bullet \quad$ Trabajo recibido el día 20 de junio de 1995

(*) Dpto. de Ingeniería Química y Metalurgia

(**) Dpto. de Geología. Universidad de Sonora. Aptdo. postal 106. Hemosillo, Sonora 83000 (México).
}

la recuperación de metales preciosos (1), lodos y polvos de las plantas de producción de ácido sulfúrico (2) y polvos de plantas de fundición de cobre y de plomo. La disponibilidad del teluro, sin embargo, está determinada por la producción de los metales a los que frecuentemente está asociado. Casi todo el teluro comercial, en consecuencia, se produce a partir de lodos electrolíticos de las refinerías de cobre que contienen desde trazas hasta un $4 \%$. En aquellas refinerías donde se recupera el teluro, se obtienen, aproximadamente, $0,065 \mathrm{~kg}$ Te/t de cobre refinado (3).

Por lo que respecta a la producción o beneficio del teluro en México, no se conoce que se haya llevado a cabo hasta la fecha; los datos oficiales no consignan si el teluro se recupera de las fundiciones o refinerías existentes.

Este trabajo presenta una revisión sobre las principales localizaciones con presencia de teluro en Sonora (México). Asimismo, se discuten los principales procesos de lixiviación y recuperación propuestos para minerales de teluro asociados a metales preciosos. 


\section{LOCALIZACIONES DE MINERALES DE TELURO EN SONORA}

Durante más de 50 años, la única localización conocida de minerales de teluro en México fue la mina La Bambolla, en las inmediaciones del poblado de Moctezuma, Sonora. En 1989 se registró la segunda localización en el yacimiento de San Francisco, $145 \mathrm{~km}$ al norte de Hermosillo (4 y 5) (Fig. 1).

La mina La Bambolla se encuentra emplazada en una secuencia de rocas volcánicas de composición andesítico-riolítica del Terciario inferior. Fue descubierta en 1936 y, entre este año y 1945 se produjeron $2.900 \mathrm{t}$ de mineral de 10 a $20 \mathrm{~kg}$ de Te/t $(6$ y 7). Valores representativos de las Minas de San Miguel, Quién sabe y La Bambolla dan concentraciones de 4-12 g Au/t, 6-664 g Ag/t y 576-3.400 g $\mathrm{Te} / \mathrm{t}(8)$.

En La Bambolla existen por lo menos cinco vetas orientadas en dirección N50-90W inclinadas 85 a $90^{\circ}$ al NE (6 y 7$)$.

Uno de los aspectos más sensacionales es la variedad mineralógica de minerales de teluro descubierta en La Bambolla, donde se han descrito por

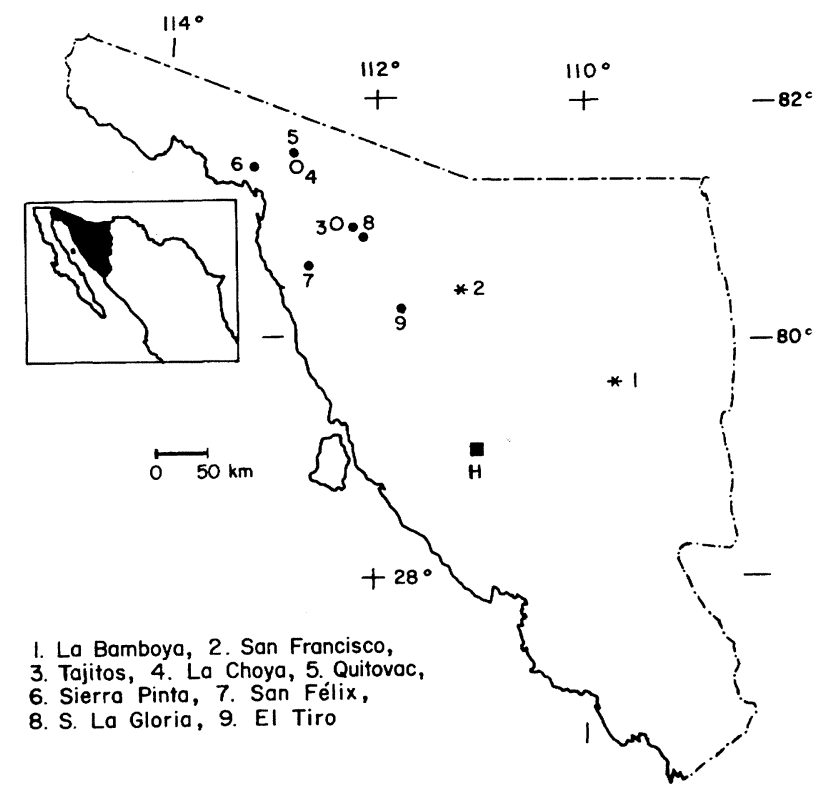

FIG. 1.- Yacimientos de interés por teluro en Sonora.

(*) Localidades con minerales de teluro.

(o) Localidades con anomalías de teluro.

$(\bullet)$ Localidades con posibles anomalías de teluro. H: Hermosillo.

FIG. 1.- Main deposits for tellurium minerals in Sonora.

(*) Localities with tellurium minerals.

(o) Localities with tellurium anomalies.

(•) Localities with possible tellurium anomalies. H: Hermosillo primera vez más de veinte especies nuevas de teluritos y teluratos (Tabla I).

Por otra parte, recientemente se informó sobre una segunda paragénesis en el yacimiento de San Francisco, muy rica también en minerales de teluro (4 y 5). En este yacimiento se han ubicado $4,28 \cdot 10^{6} \mathrm{t}$ de mineral con una ley de $1,76 \mathrm{~g} \mathrm{Au} / \mathrm{t}$ (9). La mineralización se asocia a un granito leucocrático cuya

TABla I.- Principales especies mineralógicas de minerales de teluro en Sonora (México)

TABLE I.-Main tellurium mineralogical species in Sonora (México)

\begin{tabular}{|c|c|c|}
\hline Mineral & Composición & Ref. \\
\hline Paratelurita* & $\mathrm{TeO}_{2}$ & (37) \\
\hline Denningita $^{*}$ & $\begin{array}{l}(\mathrm{Ca}, \mathrm{Mn})(\mathrm{Mn}, \mathrm{Zn}) \\
\mathrm{Te}_{4} \mathrm{O}_{10}\end{array}$ & $(38)$ \\
\hline Spiroffita* & $(\mathrm{Mn}, \mathrm{Zn})_{2} \mathrm{Te}_{3} \mathrm{O}_{8}$ & (24) \\
\hline Petzita* & $\mathrm{Ag}_{3} \mathrm{AuTe}_{2}$ & $(4$ y 5$)$ \\
\hline Silvanita** & $\mathrm{AgAuTe}_{4}$ & $(4$ y 5$)$ \\
\hline Calaverita** & $\mathrm{AuTe}_{2}$ & $(4$ y 5$)$ \\
\hline Altaíta** & $\mathrm{PbTe}$ & $(4$ y 5$)$ \\
\hline San Francisco $1^{* *}$ & $\mathrm{Te}_{5}(\mathrm{~Pb}, \mathrm{Bi})_{4}$ & $(4$ y 5$)$ \\
\hline San Francisco $2 * *$ & $\mathrm{TeAgS}_{3}$ & $(4$ y 5$)$ \\
\hline Blackeita* & $\mathrm{Fe}, \mathrm{TeO}_{3}(?)$ & $(6$ y 7$)$ \\
\hline Emmonsita* & $\mathrm{Fe}_{2}\left(\mathrm{TeO}_{3}\right)_{3} \cdot 2 \mathrm{H}_{2} \mathrm{O}$ & $(6$ y 7$)$ \\
\hline Moctezumita* & $\mathrm{Pb}\left(\mathrm{UO}_{2}\right)\left(\mathrm{TeO}_{3}\right)_{2}$ & $(6$ y 7$)$ \\
\hline Telurita* & $\mathrm{TeO}_{2}$ & $(4-7)$ \\
\hline Teluro nativo*,** & $\mathrm{Te}$ & $(6$ y 7$)$ \\
\hline Tetradimita* & $\mathrm{Bi}_{14} \mathrm{Te}_{13} \mathrm{~S}_{8}$ & $(6$ y 7$)$ \\
\hline Poughita* & $\mathrm{Fe}_{2}\left(\mathrm{TeO}_{3}\right)_{2} \mathrm{SO}_{4} \cdot 2 \mathrm{H}_{2} \mathrm{O}$ & $(25)$ \\
\hline Sonoraita* & $\mathrm{FeTeO}_{3}(\mathrm{OH}) \cdot \mathrm{H}_{2} \mathrm{O}$ & (39) \\
\hline Cliffordita*** & $\mathrm{UTe}_{3} \mathrm{O}_{9}$ & $(40)$ \\
\hline Zemannita* & $(\mathrm{H}, \mathrm{Na})_{2}(\mathrm{Zn}, \mathrm{Fe})_{2}\left(\mathrm{TeO}_{3}\right)_{3}$ & $(41)$ \\
\hline Schmitterita* & $\left(\mathrm{UO}_{2}\right) \mathrm{TeO}_{3}$ & $(42)$ \\
\hline Bambollaita* & $\mathrm{Cu}(\mathrm{Se}, \mathrm{Te})_{2}$ & (43) \\
\hline Hessita ${ }^{*}, * *$ & $\mathrm{Ag}_{2} \mathrm{Te}$ & $(4,5$ y 44$)$ \\
\hline Quetzalcoatlita* & $\mathrm{Cu}_{4} \mathrm{Zn}_{8}\left(\mathrm{TeO}_{3}\right)_{3}(\mathrm{OH})_{18}$ & $(20$ \\
\hline Cesbronita* $^{*}$ & $\mathrm{Cu}_{5}\left(\mathrm{TeO}_{3}\right)_{2}(\mathrm{OH})_{6} \cdot 2 \mathrm{H}_{2} \mathrm{O}$ & $(45)$ \\
\hline Carlfriessita ${ }^{*}$ & $\mathrm{CaTe}_{3} \mathrm{O}_{8}$ & $(46)$ \\
\hline Mroseita* & $\mathrm{CaTeO}_{2}\left(\mathrm{CO}_{3}\right)$ & (47) \\
\hline Burkhardita* & $\begin{array}{l}\mathrm{Pb}_{2}(\mathrm{Fe}, \mathrm{Mn}) \mathrm{Te}\left(\mathrm{Si}_{3} \mathrm{Al}\right) \mathrm{O}_{12} \\
(\mathrm{OH})_{2} \cdot \mathrm{H}_{2} \mathrm{O}\end{array}$ & $(48)$ \\
\hline Cuzticita* & $\mathrm{Fe}_{2} \mathrm{TeO}_{6} \cdot 3 \mathrm{H}_{2} \mathrm{O}$ & $(49)$ \\
\hline Eztlita* $^{*}$ & $\begin{array}{l}\mathrm{Pb}_{2} \mathrm{Fe}_{6} \mathrm{Te}_{4} \mathrm{O}_{15}(\mathrm{OH})_{10^{\circ}} \\
8 \mathrm{H}_{2} \mathrm{O}\end{array}$ & (28) \\
\hline Benleonardita* & $\mathrm{Ag}_{8}(\mathrm{Sb}, \mathrm{As}) \mathrm{Te}_{2} \mathrm{~S}_{3}$ & $(50)$ \\
\hline Cervelleita* & $\mathrm{Ag}_{4} \mathrm{TeS}$ & $(51)$ \\
\hline
\end{tabular}

* Identificado en La Bambolla.

** Identificado en San Francisco. 
sericita de alteración hidrotermal ha arrojado edades de 41 millones de años. Dicho granito se emplaza en rocas metamórficas del Precámbrico, que arrojan una edad mínima de metamorfismo de 911 $\pm 9,2$ millones de años obtenida sobre anfíbolas (10). La mineralización está en vetas de cuarzo lechoso y turmalina (chorlita y dravita), con menores cantidades de albita, sericita, clorita y calcita. La paragénesis metálica se caracteriza por una asociación de Au-Te con contenidos anómalos de plomo, molibdeno, bismuto y volframio. Los minerales reconocidos, aparte del oro nativo, son: petzita, hessita, silvanita, calaverita, teluro nativo, altaíta y otros dos llamados informalmente "San Francisco 1" $\left(\mathrm{Te}_{5}(\mathrm{~Pb}, \mathrm{Bi})_{4}\right)$ y "San Fancisco 2" $\left(\mathrm{TeAgS}_{3}\right)$. Otros minerales existentes son pirita, galena y molibdenita, en la zona primaria, y wulfenita en la zona de oxidación.

A pesar de que se han reconocido otras localizaciones con anomalías de teluro y otras más donde se presupone su existencia (Fig. 1), la única explotación histórica ha sido La Bambolla.

\section{POSIBILIDADES DE EXPLORACIÓN}

Las dos localizaciones antes mencionadas son las únicas que se conocen hasta hoy en México, pero seguramente deben existir otras no descubiertas. Por ejemplo, McAnulty (11) menciona la existencia de mineralizaciones de teluro en un área de $100 \mathrm{~km}^{2}$ en la Sierra Blanca, cerca de Moctezuma. Por otra parte, en el área de Tajitos, al Noroeste de Sonora existen vetas de cuarzo con oro, encajonadas en andesitas y riolitas jurásicas. Datos geocronométricos de sericita de las vetas consignan una edad de 49,2 \pm 2 millones de años (12). Análisis geoquímicos representativos de las vetas de Tajitos tienen valores de teluro entre 1 y $105 \mathrm{~g} / \mathrm{t}$; estos valores son notablemente superiores a los que se encuentran en San Francisco. Es muy probable que un trabajo muy detallado al microscopio minerográfico y a la microsonda electrónica permita identificar minerales de teluro en Tajitos.

Un dato interesante en los dos yacimientos antes descritos es que, en el caso de La Bambolla, se trata de un depósito claramente epitermal (13), cuyas características se consideran de niveles muy someros (14), mientras que San Francisco, Tajitos y otros depósitos del desierto sonorense han sido definidos como yacimientos de tipo mesotermal (4 y 15). Otra característica distintiva es que en $L a$ Bambolla existe un sistema con un exceso de teluro en relación con el oro $(\mathrm{Te} / \mathrm{Au}<100)$, razón por la cual, a partir del mineral primario principal, que es teluro nativo, se han podido formar numerosos minerales secundarios que tienen en su composición elementos menores como plomo, zinc, uranio, cobre, etc. En San Francisco, por el contrario, la relación $\mathrm{Te} / \mathrm{Au}$ es cercana a 1 , pero la paragénesis primaria resultante en telururos, es más rica que en La Bambolla.

Paragénesis con minerales de teluro son, en efecto, muy comunes en depósitos epitermales (1618), así como en depósitos mesotermales o en shear zone (19-21). Visto en detalle, los trabajos de Markham (20 y 21) demostraron que en algunos yacimientos epitermales abunda el teluro nativo, el oro nativo es raro, la krennerita está presente y la calaverita es escasa, mientras que en los de shear zone, el teluro nativo es raro, el oro abunda, la calaverita está en exceso sobre la krennerita y están presentes telururos de mercurio, plomo, cobre y bismuto. Aun cuando la presencia o ausencia de krennerita en los depósitos no tiene relación aparente con la temperatura, las observaciones hechas por Markham (24 y 25) parecen verificarse en el caso de las manifestaciones de teluro sonorense. Por un lado, el caso de La Bambolla (un yacimiento epitermal) donde el teluro está en exceso y no se registra oro nativo ni muchos telururos, mientras que en San Francisco (un yacimiento mesotermal) el oro es abundante, hay calaverita y abundan otros telururos, aun cuando las concentraciones globales de teluro son bajas.

De acuerdo con lo anterior, yacimientos como San Francisco, Tajitos, La Choya y otros del cinturón Estación Llano-Sonoita (Fig. 1), donde se han detectado mineralizaciones o anomalías de teluro, si bien enseñan una paragénesis constante con $\mathrm{Au}(\mathrm{Pb}$ Te-W), es difícil que en ellos se puedan encontrar concentraciones económicas de teluro, ya que, asumiendo una relación $\mathrm{Au} / \mathrm{Te}$ de 1 , el valor bruto del teluro representaría un $0,1 \%$ del valor del mineral; por lo que los depósitos epitermales y, en partícular, los de la zona de Moctezuma, son los más importantes para concentraciones económicas de teluro.

A continuación, se discuten algunos de los pretratamientos o tratamientos de minerales de $\mathrm{Au}-\mathrm{Te}$ y Ag-Te, enfatizando las posibilidades de recuperar tanto el teluro como los metales preciosos.

\section{TRATAMIENTO DE TELURUROS DE METALES PRECIOSOS}

\subsection{Extracción}

Se sabe desde hace tiempo que las menas que contienen telururos de oro son más difíciles de procesar por el método convencional de cianuración. Sin embargo, existe una controversia en cuanto a la causa real que origina la refractariedad de este tipo de minerales. Por ejemplo, Haszard (26) y Johnston (27) concuerdan que si se muele finamente los telururos y se utiliza cal en exceso, se obtienen extracciones de oro alrededor del $98 \%$. Asimismo, se encontró que la velocidad de extracción se incrementaba considerablemente si se añadía peróxido 
de sodio. Otros investigadores (28 y 29), sin embargo, señalan que el oro en forma de telururo es mucho más fácil de disolver que el oro metálico.

Para el tratamiento de minerales refractarios de telururos de oro se requiere una etapa de preoxidación. Por ejemplo, en Kalgoorlie, Australia, antes de la introducción de la flotación, el mineral se oxidaba y cianuraba simultáneamente utilizando bromocianógeno: Proceso Diehl (30). En la actualidad, se utiliza la tostación del concentrado seguido por una cianuración del calcinado.

De las prácticas industriales, se sabe que Emperor, Fiji y Kalgoorlie (Australia) utilizan cloración y tostación, respectivamente, como un paso de oxidación para promover la liberación del oro antes de la cianuración. Asimismo, Emperor es aparentemente la única planta en el mundo que ha recuperado y producido teluro a partir de telururos de oro utilizando un procedimiento de lixiviación con sulfuro de sodio y precipitación con sulfito de sodio (31).

Los tratamientos de los telururos dependen de la composición química, cantidades y propiedades de los elementos asociados. Las propiedades físicas y químicas del teluro, por otro lado, determinan los métodos de extracción. Entre las propiedades más importantes se encuentran:

a) presión de vapor de los óxidos de teluro,

b) baja solubilidad de los compuestos hexavalentes en soluciones acuosas,

c) alta solubilidad de los compuestos tetravalentes en soluciones acuosas, $y$

d) facilidad para reducir compuestos solubles en soluciones acuosas con dióxido de azufre.

De acuerdo con el diagrama potencial-pH de la figura 2, el teluro puede solubilizarse en medios tanto ácidos como básicos requiriéndose la presencia de un oxidante. Este procedimiento se conoce como lixiviación directa. Otra alternativa consiste en un pretratamiento del material para convertir el teluro a su estado tetravalente o hexavalente, seguido de una lixiviación en medio ácido o básico; el procedimiento se denomina lixiviación indirecta. La figura 2 demuestra, además, que el teluro puede oxidarse, dependiendo del $\mathrm{pH}$, a ion $\mathrm{Te}^{4+}$ para soluciones extremadamente ácidas, por ejemplo, $\mathrm{pH} \sim 0$, para $0<\mathrm{pH}<2$ el ion teluril $\mathrm{HTeO}^{2+}$ mientras que para $\mathrm{pH}>2$ se produce ácido teluroso, $\mathrm{H}_{2} \mathrm{TeO}_{3}$. En soluciones básicas, la especie estable es el ion teluroso $\mathrm{TeO}_{3}^{2-}$

Las diferentes alternativas pueden describirse para teluro elemental, de acuerdo con los siguientes esquemas

Para pH ácido:

$$
\begin{aligned}
& \mathrm{Te} \rightarrow \mathrm{Te}^{4+}+4 \mathrm{e}^{-} \\
& \mathrm{Te}+2 \mathrm{H}_{2} \mathrm{O} \rightarrow \mathrm{HTeO}_{2}^{+}+3 \mathrm{H}^{+}+4 \mathrm{e}^{-}
\end{aligned}
$$

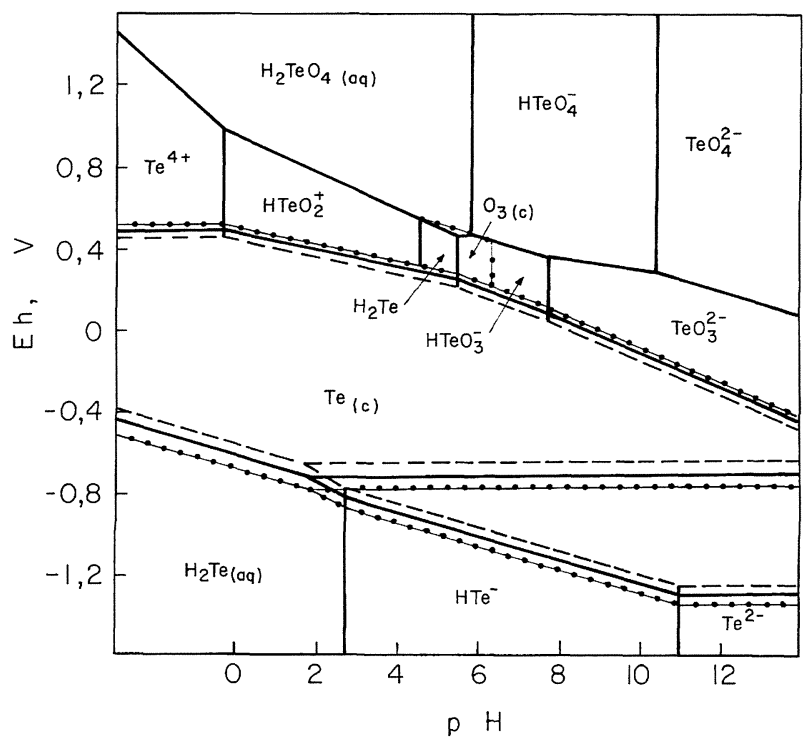

FIG. 2.- Diagrama de potencial-pH para el sistema $\mathrm{Te}-\mathrm{H}_{2} \mathrm{O}$ a $25{ }^{\circ} \mathrm{C}$. Actividades de especies de teluro en solución: .-..-- $10^{-2},-10^{-4}$ y - - - 10-6.

FIG. 2.- Potential-pH diagram for $\mathrm{Te}-\mathrm{H}_{2} \mathrm{O}$ at 25 ${ }^{\circ} \mathrm{C}$. Activities of tellurium species in solution: .....$10^{-2},-10^{-4} y-\ldots 10^{-6}$.

$$
\mathrm{Te}+3 \mathrm{H}_{2} \mathrm{O} \rightarrow \mathrm{H}_{2} \mathrm{TeO}_{3}+4 \mathrm{H}^{+}+4 \mathrm{e}^{-}
$$

Para pH básico:

$$
\mathrm{Te}+3 \mathrm{H}_{2} \mathrm{O} \rightarrow \mathrm{TeO}_{3}^{2-}+6 \mathrm{H}^{+}+4 \mathrm{e}^{-}
$$

Recientemente, Aguayo et al. (32 y 13) realizaron un estudio comparativo de la disolución de dióxido de teluro y de un concentrado de metales preciosos que contienen teluro en medio ácido y alcalino. Este concentrado se obtuvo por separación en medios pesados de un mineral oxidado de la mina La Bombolla. Se encontró que el pretratamiento ácido fue mucho más efectivo, obteniéndose cerca del $100 \%$ de extracción de teluro en aproximadamente 15 min; además, la cianuración para el concentrado pretratado resultó mejor, ya que la extracción de oro se incrementó de 66 a $95 \%$.

\subsection{Métodos alcalinos}

No se tiene conocimiento de aplicación industriales con excepción de la variante que utiliza sulfuro de sodio como agente lixiviante (15 y 27). En principio, materiales que contengan teluro elemental pueden lixiviarse en medio básico. Para el caso de telururos metálicos se recomienda la utilización de presiones superiores a la atmosférica. Para el caso de calaverita y hessita: 


$$
\begin{aligned}
& \mathrm{AuTe}_{2}+4 \mathrm{NaOH}+2 \mathrm{O}_{2} \rightarrow 2 \mathrm{Na}_{2} \mathrm{TeO}_{3}+\mathrm{Au}+\mathrm{H}_{2} \mathrm{O} \\
& \mathrm{Ag}_{2} \mathrm{Te}+2 \mathrm{NaOH}+\mathrm{O}_{2} \rightarrow \mathrm{Na}_{2} \mathrm{TeO}_{3}+2 \mathrm{Ag}+\mathrm{H}_{2} \mathrm{O}
\end{aligned}
$$

En presencia de un exceso de oxígeno puede producirse telurato, para el caso de la calaverita:

$$
\mathrm{AuTe}_{2}+4 \mathrm{NaOH}+3 \mathrm{O}_{2} \rightarrow 2 \mathrm{Na}_{2} \mathrm{TeO}_{4}+\mathrm{Au}+2 \mathrm{H}_{2} \mathrm{O}[7]
$$

El método con sulfuro es una variante del alcalino y se recomienda en aquellos casos en que el teluro se encuentra como teluro metálico, óxido o hidróxido. El teluro se extrae aparentemente a través de la formación de telurosulfuros de sodio:

$$
\begin{aligned}
& \mathrm{Na}_{2} \mathrm{~S}+\mathrm{xTe} \rightarrow \mathrm{Na}_{2} \mathrm{STe}_{\mathrm{x}} \\
& \mathrm{TeO}_{2}+\mathrm{Na}_{2} \mathrm{~S} \rightarrow \mathrm{Na}_{2} \mathrm{TeO}_{2} \mathrm{~S} \\
& \mathrm{TeO}_{2}+2 \mathrm{Na}_{2} \mathrm{~S}+\mathrm{H}_{2} \mathrm{O} \rightarrow \mathrm{Na}_{2} \mathrm{TeOS}_{2}+2 \mathrm{NaOH} \\
& \mathrm{TeO}_{2}+3 \mathrm{Na}_{2} \mathrm{~S}+2 \mathrm{H}_{2} \mathrm{O} \rightarrow \mathrm{Na}_{2} \mathrm{TeS}_{3}+4 \mathrm{NaOH} \\
& \mathrm{TeO}_{2}+5 \mathrm{Na}_{2} \mathrm{~S}+2 \mathrm{H}_{2} \mathrm{O} \rightarrow \mathrm{Na}_{6} \mathrm{TeS}_{5}+4 \mathrm{NaOH}
\end{aligned}
$$

Para el caso de soluciones alcalinas en prresencia de cianuro se produce ácido teluroso. Por ejemplo, Jayaskera (33) propone que la disolución de calaverita en medio básico, en presencia de cianuro, ocurre mediante la siguiente reacción:

$\mathrm{AuTe}_{2}+2 \mathrm{CN}^{-}+8 \mathrm{OH}^{-} \rightarrow \mathrm{Au}(\mathrm{CN})_{2}^{-}+2 \mathrm{H}_{2} \mathrm{TeO}_{3}+2 \mathrm{H}_{2} \mathrm{O}+9 \mathrm{e}^{-}$

Para condiciones típicas de cianuración, la velocidad de disolución de la calaverita es extremadamente baja, pero puede incrementarse por medio de un oxidante más enérgico como oxígeno o hipoclorito. Para este último caso, se recomienda una preoxidación, previa a la cianuración, evitando la descomposición del cianuro por medio del hipoclorito.

\subsection{Métodos ácidos}

No se tienen aplicaciones a nivel industrial utilizando este procedimiento. En términos generales, el método consiste en poner en contacto el material con un ácido, preferiblemente sulfúrico, sin o en presencia de un oxidante. Dependiendo de las condiciones, el teluro puede pasar a solución o permanecer en el sólido. En el caso de utilizar ácido sulfúrico, la reacción para el teluro metálico, la calaverita y la hessita pueden representarse por:

$$
\begin{gathered}
\mathrm{Te}+2 \mathrm{H}_{2} \mathrm{SO}_{4}+\mathrm{H}_{2} \mathrm{O} \rightarrow \mathrm{H}_{2} \mathrm{TeO}_{3}+2 \mathrm{H}_{2} \mathrm{SO}_{3} \\
\mathrm{AuTe}_{2}+4 \mathrm{H}_{2} \mathrm{SO}_{4}+2 \mathrm{H}_{2} \mathrm{O} \rightarrow 2 \mathrm{H}_{2} \mathrm{TeO}_{3}+\mathrm{Au}+4 \mathrm{H}_{2} \mathrm{SO}_{3} \\
\mathrm{Ag}_{2} \mathrm{Te}+2 \mathrm{H}_{2} \mathrm{SO}_{4}+\mathrm{H}_{2} \mathrm{O} \rightarrow \mathrm{H}_{2} \mathrm{TeO}_{3}+2 \mathrm{Ag}+\mathrm{H}_{2} \mathrm{SO}_{3}
\end{gathered}
$$

En presencia de un exceso de oxígeno, para el caso de la calaverita:

$$
\mathrm{AuTe}_{2}+2 \mathrm{H}_{2} \mathrm{SO}_{4}+2 \mathrm{O}_{2} \rightarrow 2 \mathrm{H}_{2} \mathrm{TeO}_{4}+\mathrm{Au}+2 \mathrm{SO}_{2}
$$

Jayaskera (34) encontró que en medio ácido, la calaverita se disuelve, dependiendo del $\mathrm{pH}$ y del potencial de oxidación, en ion teluril $\left(\mathrm{HTeO}_{2}^{+}\right)$o ácido teluroso $\left(\mathrm{H}_{2} \mathrm{TeO}_{3}\right)$. Este último forma una película insoluble que aparentemente inhibe la reacción a $\mathrm{pH}$ alto. Sugiere condiciones de lixiviación directa y, en el caso de medio ácido, en presencia de cloruro, se propone utilizar ion férrico como agente oxidante.

Aguayo et al. han analizado la disolución de calaverita sintética en medio ácido en presencia de tiourea como agente acomplejante (35). El comportamiento es similar al indicado por Jayaskera (27 y 33) para medio de ácidos perclórico y clohídrico. Sin embargo, en presencia de tiourea se observó que la oxidación de la calaverita es mucho más fácil, ya que se requieren potenciales de oxidación de c.a. de $0,3 \mathrm{~V}$ frente $\mathrm{ENH}$, mientras que en ausencia de agentes acomplejantes se necesitan potenciales superiores a $0,7 \mathrm{~V}$ frente ENH para lograr una disolución significativa.

\subsection{Recuperación}

Existen varios métodos para recuperar el teluro de soluciones acuosas, entre los que se pueden mencionar como más relevantes, la reducción con $\mathrm{SO}_{2}$, $\mathrm{H}_{2}$ ó $\mathrm{N}_{2} \mathrm{H}$, la cementación y la electrólisis (36).

El teluro de soluciones ácidas, se recupera regularmente a través de una reducción con $\mathrm{SO}_{2}$.

$$
\mathrm{HTeO}_{2}^{+}+2 \mathrm{SO}_{2}+2 \mathrm{H}_{2} \mathrm{O} \rightarrow \mathrm{Te}+\mathrm{H}^{+}+2 \mathrm{H}_{2} \mathrm{SO}_{4}
$$

En la reducción de teluro influyen la concentración de ácido y la temperatura. Conforme disminuyen ambas, la velocidad aumenta. Uno de los principales problemas asociados con este procedimiento se relaciona con la velocidad de asentamiento del teluro producido.

A partir de soluciones alcalinas, el teluro puede recuperarse por cementación con un metal más electronegativo, como el zinc o el aluminio:

$$
\begin{aligned}
& \mathrm{ZnO}_{2}^{2-}+4 \mathrm{H}^{+}+2 \mathrm{e}^{-} \rightarrow \mathrm{Zn}+2 \mathrm{H}_{2} \mathrm{O} ; \mathrm{E}^{\circ}=0,4412 \mathrm{~V} \\
& \mathrm{AlO}_{2}^{-}+4 \mathrm{H}^{+}+3 \mathrm{e}^{-} \rightarrow \mathrm{Al}+2 \mathrm{H}_{2} \mathrm{O} ; \mathrm{E}^{\circ}=-1,2624 \mathrm{~V} \\
& \mathrm{TeO}_{4}^{2-} \stackrel{\mathrm{E}^{\circ}=0,8975 \mathrm{~V}}{\longrightarrow} \mathrm{TeO}_{3}^{2-} \stackrel{\mathrm{E}^{\circ}=0,8269 \mathrm{~V}}{\longrightarrow} \mathrm{Te}
\end{aligned}
$$

La cementación está afectada por el tamaño de partícula y por la cantidad de agente cementante, la temperatura y la alcalinidad. 
El teluro puede recuperarse también por electrodeposición a partir de soluciones alcalinas. Los electrodos son de acero inoxidable y el depósito es un polvo de poca adherencia. El rendimiento de corriente es bajo, aunque pueden lograrse recuperaciones hasta del $99 \%$.

\section{CONCLUSIONES}

La existencia de concentraciones con minerales de teluro en Sonora se presenta en dos grandes grupos de yacimientos.

a) Depósitos epitermales, principalmente en la región de Moctezuma, donde el teluro se asocia con oro, en una relación $\mathrm{Au} / \mathrm{Te}<<1$, y donde el teluro puede llegar a representar hasta un $30 \%$ del valor del mineral, a condición de que la extracción y la recuperación sean rentables.

b) Depósitos mesotermales, hacia la provincia del desierto de Sonora, donde el teluro también se asocia con oro, pero con una relación $\mathrm{Au} / \mathrm{Te}=1$, y donde el teluro no tiene ningún interés económico salvo como guía geoquímica para la exploración de yacimientos de oro.

Generalmente, el teluro se recupera a partir de productos intermedios derivados del procesamiento de minerales de sulfuros no férreos, con excepción de un caso, industrialmente el teluro no se recupera como subproducto durante el procesamiento de metales preciosos. En este caso, se recomienda un pretratamiento para evitar la interferencia del teluro en la cianuración convencional.

Los concentrados de telururos de metales preciosos pueden procesarse por métodos acuosos que, de acuerdo con sus condiciones de trabajo, se dividen en dos grandes áreas:

a) Para $\mathrm{pH}$ ácido, aun cuando no se aplica industrialmente, presenta variantes que permiten obtener el teluro en solución o mantenerlo en el sólido. Dependiendo del rango de $\mathrm{pH}$ pueden obtenerse $\mathrm{Te}^{4+}, \mathrm{HTeO}_{2}^{+}$y $\mathrm{H}_{2} \mathrm{TeO}_{3}$.

b) Para pH básico, se ha aplicado a nivel industrial una alternativa en la que se utiliza sulfuro de sodio y se produce $\mathrm{TeO}_{3}^{2-}$. Se recomienda en aquellos casos en que el teluro se encuentra como teluro metálico, óxido o hidróxido.

El teluro en solución, dependiendo del $\mathrm{pH}$ puede recuperarse por métodos convencionales que incluyen electrodeposición, cementación y precipitación gaseosa, siendo esta última la más recomendable por su efectividad y flexibilidad.

\section{REFERENCIAS}

(1) SKInNER, P.E. Proc. 3rd. Intern. Symp. Industrial Uses of Se and Te.

(2) COOPER, W.C. (Ed.). Tellurium. Van Nostrand Teinhold Co. Nueva York, 1971.

(3) Jensen, N.L. Tellurium, en Minerals Facts and Problems. 1985. USBM Bulletin 675. Washington, D.C.

(4) Pérez Segura, E. XVIII Conv. Nacional de la Asoc. de Ing. de Minas, Metalurgistas y Geólogos de México, A.C. Memorias Técnicas, 1983: 63-79.

(5) Pérez Segura, E. The Geol. Soc. of America. Cordilleran Sect. 86th Annual Meeting. Abstracts with programs. 1990: 75 .

(6) GAINES, R.V. Bol. Inst. Geol, UNAM, 75 (1), 1965: 1-15.

(7) GAINES, R.V. Amer. Mineral., 50, 1965: 1158-1163.

(8) DeEn, J.A. y AtKinson Jr., W.W. Econ. Geol., 83, 1988: 1841-1855.

(9) Luna, R. y Gastelum, A. en Geology and Mineral Resources of Northern Sierra Madre Occidental, México. K.F. Clark, J. Roldán-Quintana y R.H. Schmidt (Eds.). El Paso Geological Soc., 1992: 307-312.

(10) Pérez-Segura, E. y Cheilletz, A. 120th AIME Annual Meeting, Denver, Co., 1991.

(11) McAnulty, W.N. The Geol. Soc. of America, Cordilleran Sect. 77th Annual Meeting. Abstracts with prrograms 13 (12), 1981: 95.

(12) Pérez-Segura, E. Contribuciones a la Tectónica del Occidente de México. L. Delgado-Argote y A. MartínBarajas (Eds.). Unión Geofísica Mexicana. Monograma núm. 1, 1993: 147-174.

(13) Encinas Romero, M.A. y Lunas Durazo, A. Universidad de Sonora. Tesis. 1991.

(14) Atkinson, W.W. III Symp. de la Geología de Sonora y Áreas Adyacentes. J. González y E.L. Vega (Eds.), 1993: 8-12.

(15) Marsden, J. y House, I. The Chemistry of Gold Exrtraction. Ellis Horwood Ltd. Londres, 1992.

(16) Casadevall, A.T. у Оhмото, H. Economic Geology 78, 1989: 521-526.

(17) Saunders, J.A. y May, E. Proc. Gold '86. Intern. Symp. Geology of Gold. A.J. MacDonald (Ed.), 1986: 436-444.

(18) Panze, J., Cruzon, G. y Watkins, Th. Colorado Mining Assoc. Mining Yearbook, 1983: 64-73.

(19) Burrows, O.R. y Spooner, T.C. Proc. Gold '86. Intern. Symp. Geology of Gold. A.T. MacDonald (Ed.) 1986: 23 29.

(20) Callan, N.J. y Spoooner, T.C., Econ. Geol. Mon., 6, 1989: 9-18.

(21) Groves, D.I., Barleya, M.E. y Ho, S.E., Econ. Geol. Mon., 6, 1989: 71-85.

(22) Markham., N.L. E con. Geol., 55 (6), 1960: 1148-1178.

(23) Markham, N.L., ibid., 55 (7), 1960: 1460-1477.

(24) Mandarino, J.A., Williams, S.J. y Mitchell, R.S. Mineral. Soc. Amer. Spec., Paper 1, 1963: 305-309.

(25) Gaines, R.V. Amer. Mineral., 53, 1968: 1075-1080. 
(26) Haszard, N.I. Proc. Australas. Inst. Min. Metall., (108), 1937: 253

(27) Cornwell, W.G. y Hisshion, R.J. Trans. AIME, 260, 1976: 108-112.

(28) DorR, J.V.N. y Bosqui, F.L. Cyanidation and Concentration of Gold and Silver Ores. McGraw-Hill, Nueva York, 1950.

(29) Hedley, N. y Tabachnick, H. Chemistry of Cyanidation. American Cyanamid Co., Mineral Dressing Notes No. 23. 1958: 1.

(30) Gowland, W. The Metallurgy of the Non-Ferrous Metals. Charles Griffin and Co. Ltd., Londres, 1921.

(31) McQuiston, F.W. y Shoemaker, R.S. Gold and Silver Cyanidation Plant Practice, vol. 1, AIME Monograph, 1975: 187

(32) Aguayo Salinas, S. y Encinas, M.A. Rev. Metal, 29 (6), 1993: 350-355.

(33) JAYASKera, S. Murdoch Univ., Western Australia, Tesis, 1989.

(34) Jayaskera, S., Ritchie, I. y Avraamides, J. World Gold '91, Cairns, 21-25 abril 1991: 181-183.

(35) Aguayo Salinas, S., Encinas R., M.A. y Gonzalez, I. IX Congreso Nacional de Electroquímica. Agosto 1994. Cuautla, México. Sdad. Mexicana de Electroquímica, 1994.

(36) Chizicov, D.M. y Shchastlivyi. Tellurium and the Tellurides. Collet's Pub., Londres, 1970
(37) Switzer, G. y Swanson, H.E. Am. Mineral., 45, 1960: $1.272-1.274$

(38) Mandarino, J.A., Williams, S.J. y Mitchel, R.S. Can. Mineral., 7, 1963: 443-452.

(39) Gaines, R.V., Donnay, G. y Hey, M.H. Am. Mineral., 53, 1968: 1.828-1.832.

(40) Gaines, R.V. Am. Mineral., 54, 1969: 697-701.

(41) Mandarino, J.A., Matzat, E. y Williams, S.J. Can. Mineral., 10, 1969: 139-140.

(42) GaInes, R.V. Am. Mineral., 56 (3-4), 1971: 411-415.

(43) Harris, D.C. y Nuffield, E.W. Can. Mineral., 11, 1972: 738-742.

(44) Williams, S.A., Miner. Mag., 39 (303), 1973: 261-263.

(45) Williams, S.A., Miner. Mag., 39 (307), 1974: 744-746.

(46) Williams, S.A. y Gaines, R.V. Miner. Mag., 40, 1975: 127-130.

(47) Mandarino, J.A., Mitchell, R.S. y Hankcock, R.G.V. Geol. Soc. Am. Abstracts with programs, 7 (6), 1975: 814-815.

(48) Gaines, R.V., Leavens, P.B. y Nelen, J.A. Am. Mineral., 64 (3-4), 1979: 355-358.

(49) Williams, S.A. Miner. Mag., 46 (339), 1982: 257-259.

(50) Stanley, C.J. Criddle, A.J. y Chisholm, J.E., Miner. Mag., 50, 1986: 681-686.

(51) Criddle, A.J., Chisholm, J.E. y Stanley, C.J. Eur. $J$. Miner., 1, 1989: 371-380. 